\title{
Prevalence and correlates of carotid plaque in a mixed HIV-serostatus cohort in Uganda
}

\author{
Prossy Bibangambah ${ }^{*}$, Linda C. Hemphill ${ }^{2,3}$, Moses Acan ${ }^{1}$, Alexander C. Tsai², Ruth N. Sentongo', \\ June-Ho Kim², Isabelle T. Yang ${ }^{4}$, Mark J. Siedner ${ }^{1,2,3}$ and Samson Okello ${ }^{1}$
}

\begin{abstract}
Background: The extent to which the risk of atherosclerotic cardiovascular disease (ACVD) is increased among people living with HIV (PLWH) in sub-Saharan Africa remains unknown.

Setting: Cross-sectional analysis nested within the Ugandan Noncommunicable Diseases and Aging Cohort, including PLWH in rural Uganda $>40$ years taking antiretroviral therapy (ART) for at least 3 years, and a population-based control group of HIV-uninfected age- and sex-matched persons.

Methods: We conducted carotid ultrasonography and collected ACVD risk factor data. Our outcome of interest was carotid plaque, defined as $>1.5 \mathrm{~mm}$ thickness from the intima-lumen interface to the media-adventitia interface. We fit multivariable logistic regression models to estimate correlates of carotid plaque including HIV-specific and traditional cardiovascular risk factors.

Results: We enrolled 155 (50.2\%) PLWH and 154 (49.8\%) HIV-uninfected comparators, with a mean age of 51.4 years. Among PLWH, the median CD4 count was 433 cells $/ \mathrm{mm}^{3}$ and $97.4 \%$ were virologically suppressed. Carotid plaque prevalence was higher among PLWH (8.4\% vs 3.3\%). HIV infection (aOR 3.90; 95\% Cl 1.12-13.60) and current smokers (aOR 6.60; 95\% Cl 1.22-35.80) had higher odds of carotid plaque, whereas moderate (aOR $0.13,95 \% \mathrm{Cl} 0.01-1.55$ ) and vigorous intensity of physical activity (aOR $0.34,95 \% \mathrm{Cl} 0.07-1.52$ ) were associated with decreased odds of carotid plaque.

Conclusion: In rural Uganda, PLWH have higher prevalence of carotid plaque compared to age- and sex-matched HIV-uninfected comparators. Future work should explore how biomedical and lifestyle modifications might reduce atherosclerotic burden among PLWH in the region.
\end{abstract}

Keywords: HIV, Carotid intima media thickness, Carotid plaque, Atherosclerosis, Cardiovascular disease

\section{Background}

There are few data on the epidemiology of atherosclerosis among PLWH in sub-Saharan Africa, which is home to at least $70 \%$ of the global burden of HIV [1]. Most of the data available from this region has focused primarily on the epidemiology of traditional risk factors of

\footnotetext{
*Correspondence: pbibangambah@must.ac.ug

${ }^{1}$ Department of Radiology, Mbarara University of Science

and Technology, P.O. Box, 1410 Mbarara, Uganda

Full list of author information is available at the end of the article
}

atherosclerosis or its clinical manifestations [2-5]. The few studies directly measuring atherosclerosis among PLWH in sub-Saharan Africa have shown conflicting results with some studies describing an increased burden of atherosclerosis among PLWH [6], others no difference [7] and others a reversal [8].

We sought to respond to this gap by estimating the prevalence of carotid plaque in a Ugandan cohort of PLWH and age- and sex-matched HIV-uninfected comparators. To do so, we conducted carotid ultrasound to detect carotid plaque, a validated surrogate marker and 
predictor of atherosclerotic CVD risk [9, 10]. Previous studies have shown that there was no difference in carotid intima media thickness (cIMT), another predictor of atherosclerotic CVD risk, between PLWH and uninfected comparators [11, 12]. Other cohorts, however, like the MultiCenter AIDS Cohort Stuudy (MACS) in the US have shown that while there was no difference in cIMT, there was a difference in carotid plaque [13] and this remains unstudied in Uganda. We hypothesized that the prevalence of carotid plaque would be higher among the PLWH compared with their HIV-uninfected comparators.

\section{Methods}

\section{Study population}

We conducted a cross-sectional within the Ugandan Noncommunicable Diseases and Aging Cohort (UGANDAC) study (NCT02445079) $[14,15]$. Briefly, UGANDAC was a prospective cohort of ambulatory PLWH aged at least 40 years on ART for at least 3 years, and a matched population-based comparator group of uninfected persons. The PLWH were enrolled as a convenience sample of HIV-infected persons receiving care at the Mbarara Regional Referral Hospital HIV clinic. We then used a complete population census study in a nearby cluster of villages [16] to identify and conduct homebased enrollment of sex-matched and age-similar (by quartile of the HIV-infected sub-group) HIV-uninfected comparators, who underwent confirmatory HIV testing prior to enrollment.

\section{Carotid ultrasonography}

All study participants underwent carotid ultrasonography at the enrollment visit. Two operators (JHK and PB) trained in carotid ultrasonography at the University of Wisconsin, USA $[17,18]$ and blinded to participant information individually collected images using a $13-6 \mathrm{MHz}$ linear transducer (Sonosite M-Turbo, Sonosite, Bothell, Washington, USA). We used a standardized carotid ultrasound scanning protocol to collect bilateral common carotid artery images in a longitudinal view from the anterior, lateral, and posterior positions [17]. We used validated semi-automated software (SonoCalc 5.0, SonoSite) [19] to measure carotid plaque, which was defined as a focal thickness of $1.5 \mathrm{~mm}$ or more protruding into the arterial lumen from the media-adventitia interface to the intima-lumen interface of the common carotid artery $[17,20]$. A board-certified cardiologist (LH) reviewed image interpretation for all plaque measurements.

\section{Covariate data collection}

The data used in this analysis were collected from participants at their enrollment study visit. Participants completed questionnaires on sociodemographic characteristics, physical activity, smoking history and history of medications including statin use. We measured weight using standardized scales (seca 762, Hanover, USA) and height using roll-up measuring stadiometers (seca 206, Hanover, USA).

We also collected resting blood pressure using digital sphygmomanometers (Omron Healthcare Inc., Bannockburn, USA). We collected blood samples for hemoglobin A1c testing (Siemens Vantage, Siemens Healthcare Diagnostics, Tarrytown, NY, USA) and lipid profile assessments (cryopreserved specimens at the Laboratory for Clinical Biochemistry Research at the University of Vermont, USA). Among PLWH, we abstracted CD4+ T-cell count and HIV-1 RNA viral load data from medical records.

\section{Study definitions and statistical analysis}

We calculated the body mass index (BMI) as weight (in kilograms) divided by the square of height (in meters) and categorized it as underweight/low if less than $18.5 \mathrm{~kg} / \mathrm{m}^{2}$, normal if between $18.5-25 \mathrm{~kg} / \mathrm{m}^{2}$, overweight if between $25-29.9 \mathrm{~kg} / \mathrm{m}^{2}$, and obese if $30 \mathrm{~kg} / \mathrm{m}^{2}$ or more [21]. Self-reported smoking status was categorized as current, former (someone who quit smoking more than twelve months prior to enrollment), and never smoker. We defined physical activity using the physical activity categories determined by the International Physical Activity Questionnaire Protocol [22]. We defined virologic suppression as having less than 1000 copies of HIV per milliliter of blood [23].

\section{Statistical analysis}

We used descriptive statistics to describe clinical and demographic characteristics for the total cohort and stratified by HIV serostatus. We graphically depicted prevalence of carotid plaque, stratified by HIV status and age (categorized as $40-49,50-59$ and 60 or more years). To estimate unadjusted and adjusted correlates of carotid plaque, we fit univariable and multivariable logistic regression models, including HIV serostatus as the primary explanatory variable of interest, and additional CVD risk factors, including sex, age, mean diastolic blood pressure, mean systolic blood pressure, glycated hemoglobin (\%HbA1c), physical activity, total cholesterol, smoking status and BMI. A p-value of $<0.05$ was considered statistically significant.

We conducted data analysis with Stata version 15.0 (StataCorp, College Station, Texas) and graphically presented prevalence of carotid plaque using Microsoft Excel 2016 (Microsoft Corporation, Washington). 


\section{Ethical consideration}

The UGANDAC study procedures were approved by the human subjects review boards at the Mbarara University of Science and Technology (Ref. 06/04-14), Partners Healthcare (Ref. 2014P001928/MGH), and the Uganda National Council for Science and Technology (Ref. HS1689). All participants provided written informed consent.

\section{Results}

We enrolled 309 study participants: 155 (50.2\%) were PLWH and 154 (49.8\%) were HIV-uninfected. As expected based on the study design, mean age and sex were similar by HIV serostatus (Table 1). Among PLWH, the median CD4 at enrollment count was 433 (IQR, 336,559) cells $/ \mathrm{ml}$ and $97.4 \%$ were virologically suppressed. About $64 \%$ were taking a nevirapine (NVP)-based ART regimen, $27.1 \%$ taking an efavirenz
(EFV)-based ART, and 9.0\% taking Protease inhibitors (Table 1). There was no statin use amongst study participants. Compared with the uninfected comparators, PLWH had lower systolic blood pressure $(112.5 \mathrm{mmHg}$ vs $117.5 \mathrm{mmHg} P=0.015)$ and lower diastolic blood pressure (69 $\mathrm{mmHg}$ vs $77 \mathrm{mmHg} ; P<0.001$ ), and were less likely to be current smokers $(5.8 \%$ [9/155] vs $20.8 \%$ [32/154]; $P<0.001)$.

The prevalence of carotid plaque was 5.8\% (95\% confidence interval [CI] 3.7-9.1) in the total cohort. PLWH had a higher prevalence than HIV uninfected comparators $(8.4 \%$ vs $3.3 \%)$. The prevalence of carotid plaque was highest among those aged 60 years and over, and tended to be higher among PLWH in all age groups but these estimates were not statistically significant (Fig. 1).

In multivariable regression models (Table 2), HIVinfection (aOR 3.90; 95\% CI 1.12-13.60, $P=0.033$ ) and

Table 1 Study participant characteristics at Enrollment into a Mixed HIV-Serostatus Cohort in Uganda

\begin{tabular}{|c|c|c|c|c|}
\hline Characteristic & Overall $(n=309)$ & HIV-negative $(n=154)$ & PLWH $(n=155)$ & $P$-value* \\
\hline Age, years (mean, SD) & $51.4(7.2)$ & $51.5(7.7)$ & $51.2(6.6)$ & 0.695 \\
\hline Female $(n, \%)$ & $151(48.9)$ & $77(50.0)$ & $74(47.7)$ & 0.691 \\
\hline Smoking status (n, \%) & & & & $<0.001$ \\
\hline Never-smoker & $163(52.8)$ & $72(46.8)$ & $91(58.7)$ & \\
\hline Former smoker & $105(34.0)$ & $50(32.5)$ & $55(35.5)$ & \\
\hline Current smoker & $41(13.3)$ & $32(20.8)$ & $9(5.8)$ & \\
\hline Physical activity $^{\beta}(\mathrm{n}, \%)$ & & & & 0.003 \\
\hline Low intensity & $16(5.2)$ & $3(2.0)$ & $13(8.4)$ & \\
\hline Moderate intensity & $42(13.6)$ & $15(9.7)$ & $27(17.4)$ & \\
\hline Vigorous intensity & $251(81.2)$ & $136(88.3)$ & $115(74.2)$ & \\
\hline Body mass index $(n, \%)$ & & & & 0.056 \\
\hline Underweight $(<18.5$ kg/m²) & $40(12.9)$ & $27(17.5)$ & $13(8.4)$ & \\
\hline Normal $\left(18.5-\leq 25 \mathrm{~kg} / \mathrm{m}^{2}\right)$ & $189(61.2)$ & $90(58.4)$ & $99(63.9)$ & \\
\hline Overweight/Obese (> 25 kg/m²) & $80(25.9)$ & $37(24.0)$ & $43(27.7)$ & \\
\hline Hemoglobin A1c, \% (mean, SD) & $5.5(1.0)$ & $5.6(0.8)$ & $5.4(1.1)$ & 0.287 \\
\hline Total cholesterol, mg/dL (mean, SD) & $160.1(35.9)$ & $159.5(33.2)$ & $160.6(38.4)$ & 0.788 \\
\hline Mean systolic BP, mmHg (median, IQR) & $114.5(105.5,126.5)$ & $117.5(108,131.5)$ & $112.5(100,120)$ & 0.015 \\
\hline Mean diastolic BP, mmHg (median, IQR) & $73(66,81.5)$ & $77(68.5,83.5)$ & $69(63.5,79.0)$ & $<0.001$ \\
\hline Virologic suppression ${ }^{\Omega}$ at enrollment (n, \%) & N/A & $\mathrm{N} / \mathrm{A}$ & $150(97.4)$ & \\
\hline Nadir CD4 count, cells/M (median, IQR) & $\mathrm{N} / \mathrm{A}$ & $\mathrm{N} / \mathrm{A}$ & $118(74,183)$ & \\
\hline CD4 count at enrollment, cells/ $\mu$ (median, IQR) & $\mathrm{N} / \mathrm{A}$ & $\mathrm{N} / \mathrm{A}$ & $433(336,559)$ & \\
\hline ART Regimen (n, \%) & $\mathrm{N} / \mathrm{A}$ & $\mathrm{N} / \mathrm{A}$ & & \\
\hline NVP-based & $\mathrm{N} / \mathrm{A}$ & $\mathrm{N} / \mathrm{A}$ & 99 (63.9) & \\
\hline EFV-based & $\mathrm{N} / \mathrm{A}$ & $\mathrm{N} / \mathrm{A}$ & $42(27.1)$ & \\
\hline Pl-based & N/A & $\mathrm{N} / \mathrm{A}$ & $14(9.0)$ & \\
\hline
\end{tabular}

IQR interquartile range; $S D$ standard deviation; $A R T$ regimen antiretroviral therapy regimen; $A Z T$ zidovudine; $3 T C$ lamivudine; NVP nevirapine; $E F V$ efavirenze; TDF tenofovir; PI protease inhibitor; $N / A$ not applicable

* $P$-values comparing the PLWH with the HIV-uninfected were calculated using chi-squared testing for categorical variables and $t$ tests for normally distributed continuous variables

${ }^{\beta}$ Physical activity categories were determined by the International Physical Activity Questionnaire (IPAQ) scoring protocol

$\Omega$ Virologic suppression defined as having less than 1000 copies of HIV per milliliter of blood 


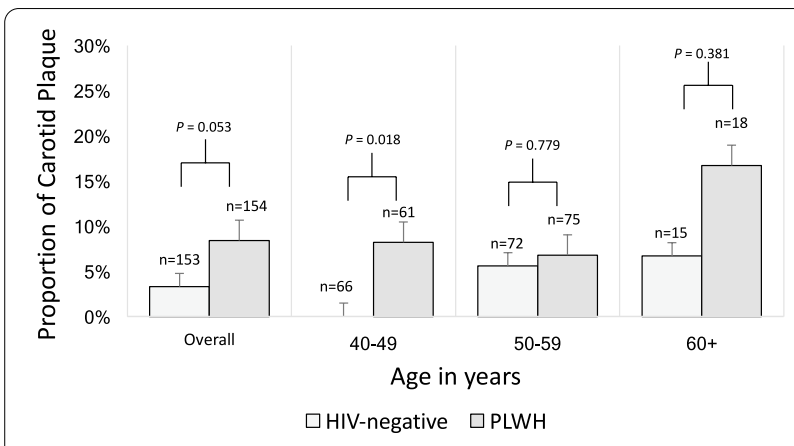

Fig. 1 Plaque prevalence by age (years) and serostatus in a Mixed HIV-serostatus cohort in Uganda

current smoking status (aOR 6.60 for current versus never smokers; 95\% CI 1.22-35.80, $P=0.029)$ were significantly associated with higher odds of carotid plaque. We also found that moderate (aOR 0.13, 95\% CI $0.01-1.55, P=0.107)$ and vigorous physical activity (aOR 0.34, 95\% CI 0.07-1.52, $P=0.156$ ) were associated with decreased odds of carotid plaque, but these estimated associations were not statistically significant.

\section{Discussion}

In this mixed HIV cohort in rural Uganda, we found the prevalence of carotid plaque was higher among PLWH compared with their HIV-uninfected counterparts. These findings align with previous studies, which have reported a higher prevalence of carotid plaque among PLWH compared with HIV-uninfected comparators [7, 13, 24-27]. For instance, a combined analsysis from the Women's Interagency HIV Study and Multicenter AIDS Cohort Study reported that PLWH had a $61 \%$ greater risk of new focal carotid artery plaque formation over seven years compared with uninfected individuals, regardless of baseline vascular phenotype and after adjusting for cardiometabolic risk factors [13]. Importantly, the relationship between HIV and the presence and formation of plaque in this literature has been consistently observed even after adjustment for other correlates of atherosclerosis. While the mechanistic details will require further study, the increased burden of carotid plaque among PLWH has been hypothesized to be mediated by persistent immune activation and inflammation that occurs in HIV infection $[14,28,29]$.

Our reported prevalence of carotid plaque is lower than that found in other studies done within sub-Saharan Africa [30]. The difference in these findings could be due to the study populations, for example, an inclusion

Table 2 Correlates of Carotid Plaque in a Mixed HIV-Serostatus Cohort in Uganda

\begin{tabular}{|c|c|c|c|c|}
\hline \multirow[t]{2}{*}{ Characteristic } & \multicolumn{2}{|l|}{ Unadjusted model } & \multicolumn{2}{|l|}{ Adjusted model } \\
\hline & Odds Ratio $(95 \% \mathrm{Cl})$ & $P$-value & Odds Ratio (95\% Cl) & $P$-value \\
\hline \multicolumn{5}{|l|}{ HIV Serostatus } \\
\hline HIV-uninfected & REF & REF & REF & REF \\
\hline PLWH & $2.73(0.95,7.85)$ & 0.063 & $3.90(1.12,13.60)$ & 0.033 \\
\hline Age (each year) & $1.04(0.99,1.10)$ & 0.146 & $1.04(0.97,1.12)$ & 0.268 \\
\hline Mean systolic BP (each mmHg increase) & $1.02(0.99,1.04)$ & 0.183 & $1.01(0.96,1.06)$ & 0.831 \\
\hline Mean diastolic BP (each mmHg increase) & $1.02(0.00,0.39)$ & 0.446 & $1.04(0.95,1.13)$ & 0.425 \\
\hline \multicolumn{5}{|l|}{ Physical Activity } \\
\hline Low & REF & REF & REF & REF \\
\hline Moderate & $0.11(0.10,1.11)$ & 0.061 & $0.13(0.01,1.55)$ & 0.107 \\
\hline Vigorous & $0.26(0.07,1.00)$ & 0.051 & $0.34(0.07,1.52)$ & 0.156 \\
\hline \multicolumn{5}{|l|}{ Smoking status } \\
\hline Never-smoker & REF & REF & REF & REF \\
\hline Current smoker & $2.83(0.76,10.54)$ & 0.121 & $6.60(1.22,35.80)$ & 0.029 \\
\hline Former smoker & $2.16(0.73,6.41)$ & 0.166 & $2.37(0.68,8.30)$ & 0.176 \\
\hline Female & $0.65(0.25,1.72)$ & 0.386 & $0.90(0.27,2.92)$ & 0.855 \\
\hline \multicolumn{5}{|l|}{ Body mass index, kg/m² } \\
\hline Normal & REF & REF & REF & REF \\
\hline Underweight & $0.35(0.04,2.73)$ & 0.315 & $0.26(0.03,2.25)$ & 0.221 \\
\hline Overweight/Obese & $0.71(0.23,2.26)$ & 0.564 & $0.85(0.20,3.58)$ & 0.823 \\
\hline Total cholesterol (each mg/dL increase) & $0.99(0.98,1.01)$ & 0.583 & $0.99(0.98,1.01)$ & 0.513 \\
\hline Hemoglobin A1c & $0.67(0.28,1.57)$ & 0.347 & $0.77(0.31,1.88)$ & 0.564 \\
\hline
\end{tabular}


criteria from a similar study in Malawi was presentation of an acute stroke-like syndrome, whereas our study population was comprised of asymptomatic participants in ambulatory care. We found higher odds of carotid plaque among those who were current smokers and a lower odds among those with moderate or vigorous physical activity. These data corroborate studies that have described physical inactivity and smoking as independent predictors of carotid plaque development [31-34]. Although we found that the prevalence of carotid plaque was higher among those aged 60 and older, we did not find significant associations between age and carotid plaque, possibly due to the relatively narrow age distribution in our study. This finding differs from other studies which have found that age has been associated with risk of carotid plaque [27].

Notably, we previously demonstrated no association in this cohort between HIV serostatus and cIMT thickness or progression [11]. While both cIMT and carotid plaque are considered predictors of atherosclerotic disease events, they vary in pathophysiology whereby cIMT represents intimal medial thickening with very early atherosclerotic changes in the intima but also, potentially, smooth muscle hypertrophy and/or hyperplasia in the media which is more related to hypertension. Alternatively, carotid plaque is predominantly intimal thickening with foam cells, smooth muscle cells, macrophages, a lipid core, and a fibrous cap depending on the age of the plaque [10]. This variation in pathophysiology could explain the difference in findings between these two modalities. It is also in keeping with studies from the United States, for example the MACS study, that similarly demonstrated greater incidence of carotid plaques in PLWH compared to HIV-uninfected, comparators but not with cIMT [13].

There is scarcity of policies that would support the integration of CVD care and coverage amongst PLWH in the region. The primary care guidelines for HIV/AIDS have been largely successful, yet integrated care for screening, diagnosis and management of CVD in this population is still lacking. Our data responds to the gap in the knowledge of the epidemiology of CVD risk that could stand as a foundation for further studies that develop the policies that could improve upon this integrated care. This would advance the healthcare delivery system, not only in rural Uganda, but also sub-Saharan Africa.

Our study was strengthened by the use of a well-characterised study cohort. However, we are unable to extrapolate beyond correlations or comment on progression or incidence of plaque due to our cross-sectional design. There could also be residual or unmeasured confounding from factors not included in our model that account for the increased prevalence seen among PLWH. This study was also limited by dichotomous measure of plaque.
Prior studies have shown that continuous measures of plaque (e.g. carotid plaque area) are also strong predictors of CVD and can be used in targeting preventive therapy [35-37]. Finally, our study might be under-powered to demonstrate smaller effect sizes.

\section{Conclusion}

In conclusion, we found a higher odds of carotid plaque among PLWH and smokers in rural southwestern Uganda. These results suggest a combination of HIVspecific and traditional risk factors might contribute to atherosclerosis in this population. Future work should explore the mechanisms underlying this observation, and determine whether lifestyle factors or other targeted interventions might reduce atherosclerotic burden among PLWH in the region.

\section{Abbreviations}

BMI: Body mass index; Cl: Confidence Interval; CIMT: Carotid intima media thickness; CVD: Cardiovascular disease; HBA1c: Glycated hemoglobin; MACS: Multicenter AIDS Cohort Study; PLWH: People living with HIV; aOR: Adjusted odds ratio; UGANDAC: Ugandan Noncommunicable Diseases and Aging Cohort study.

\section{Acknowledgements \\ The authors are extremely grateful for the support and partnership of the study participants and staff of the UGANDAC study.}

\section{Authors' contributions}

MS, SO, PB and MA did the study concept and design. PB, JK, RS and SO acquired the data. PB, MS and SO carried out the data analysis and interpretation. $\mathrm{PB}, \mathrm{MS}$ and $\mathrm{SO}$ drafted the manuscript. Critical revision of the manuscript was done by PB, LH, IY, JK, RS, MA, AT, SO and MS. All authors read and approved the final manuscript.

\section{Funding}

This work was supported by the Fogarty International Center, National Institute of Mental Health, of the National Institutes of Health under Award Number D43 TW010543, and National Institute of Diabetes and Digestive and Kidney Diseases of the National Institutes of Health. The content is solely the responsibility of the authors and does not necessarily represent the official views of the National Institutes of Health. The UGANDAC study was funded by the National Institutes of Health (R21 HL124712, P30 Al060354, R24 AG044325, P30 AG024409, P30 Al027763, R01 HL141053, and R01 MH113494), and the Massachusetts General Hospital Executive Committee on Research.

Availability of data and materials

The datasets used and/or analysed during the current study are available from the corresponding author on reasonable request.

\section{Declarations}

\section{Ethics approval and consent to participate}

This research was performed in accordance with the Declaration of Helsinki. The UGANDAC study procedures were reviewed and approved by the human subjects review boards at the Mbarara University of Science and Technology (Ref. 06/04-14), Partners Healthcare (Ref. 2014P001928/MGH), and the Uganda National Council for Science and Technology (Ref. HS1689). All participants provided written informed consent.

Consent for publication

Not applicable. 


\section{Competing interests}

$\mathrm{LH}$ receives research support from Regeneron and Novartis. The remaining authors declare that they have no competing interests.

\section{Author details}

${ }^{1}$ Department of Radiology, Mbarara University of Science and Technology, P.O. Box, 1410 Mbarara, Uganda. ${ }^{2}$ Massachusetts General Hospital, Boston, MA, USA. ${ }^{3}$ Harvard Medical School, Boston, MA, USA. ${ }^{4}$ Geisel School of Medicine at Dartmouth, Hanover, NH, USA.

\section{Received: 26 October 2021 Accepted: 7 December 2021}

\section{Published online: 15 December 2021}

\section{References}

1. Kharsany ABM, Karim QA. HIV infection and AIDS in Sub-Saharan Africa: current status challenges and opportunities. Open AIDS J. 2016;10:34-48.

2. Mensah GA. Ischaemic heart disease in Africa. Heart Br Card Soc. 2008;94(7):836-43.

3. Levitt NS. Diabetes in Africa: epidemiology, management and healthcare challenges. Heart. 2008;94(11):1376-82.

4. Okello S, Amir A, Bloomfield GS, Kentoffio K, Lugobe HM, Reynolds Z, et al. Prevention of cardiovascular disease among people living with HIV in sub-Saharan Africa. Prog Cardiovasc Dis. 2020;63(2):149-59.

5. Imoisili OE, Sumner AE. Preventing diabetes and atherosclerosis in sub-Saharan Africa: Should the metabolic syndrome have a role? Curr Cardiovasc Risk Rep. 2009:3(3):161-7.

6. Vos AG, Barth RE, Klipstein-Grobusch K, Tempelman HA, Devillé WL, Dodd C, et al. Cardiovascular Disease Burden in Rural Africa: Does HIV and Antiretroviral Treatment Play a Role? J Am Heart Assoc Cardiovasc Cerebrovasc Dis [Internet]. 2020 Mar 30;9(7). Available from: https://www. ncbi.nlm.nih.gov/pmc/articles/PMC7428654/

7. Mosepele M, Hemphill LC, Moloi W, Moyo S, Nkele I, Makhema J, et al. Pre-clinical carotid atherosclerosis and SCD163 among virally suppressed HIV patients in Botswana compared with uninfected controls. PLoS ONE [Internet]. 2017 Jun 29;12(6). Available from: https://www.ncbi.nIm.nih. gov/pmc/articles/PMC5491105/

8. Fourie CMT, Schutte AE, Smith W, Kruger A, van Rooyen JM. Endothelial activation and cardiometabolic profiles of treated and never-treated HIV infected Africans. Atherosclerosis. 2015;240(1):154-60.

9. Inaba Y, Chen JA, Bergmann SR. Carotid plaque, compared with carotid intima-media thickness, more accurately predicts coronary artery disease events: a meta-analysis. Atherosclerosis. 2012;220(1):128-33.

10. Naqvi TZ, Lee M-S. Carotid intima-media thickness and plaque in cardiovascular risk assessment. JACC Cardiovasc Imaging. 2014;7(10):1025-38.

11. Siedner MJ, Bibangambah P, Kim J, Lankowski A, Chang JL, Yang IT, et al. Treated HIV infection and progression of carotid atherosclerosis in rural Uganda: a prospective observational cohort study. J Am Heart Assoc. 2021:10(12):9994.

12. Sarfo FS, Nichols M, Agyei B, Singh A, Ennin E, Nyantakyi AD, et al. Burden of subclinical carotid atherosclerosis and vascular risk factors among people living with HIV in Ghana. J Neurol Sci. 2019;15(397):103-11.

13. Hanna DB, Post WS, Deal JA, Hodis HN, Jacobson LP, Mack WJ, et al. HIV infection is associated with progression of subclinical carotid atherosclerosis. Clin Infect Dis. 2015;61(4):640-50.

14. Siedner MJ, Kim J-H, Nakku RS, Bibangambah P, Hemphill L, Triant VA, et al. Persistent immune activation and carotid atherosclerosis in HIV-Infected Ugandans receiving antiretroviral therapy. J Infect Dis. 2016;213(3):370-8.

15. Siedner MJ, Kim J-H, Nakku RS, Hemphill L, Triant VA, Haberer JE, et al. HIV infection and arterial stiffness among older-adults taking antiretroviral therapy in rural Uganda. AIDS Lond Engl. 2016:30(4):667-70.

16. Takada S, Nyakato V, Nishi A, O'Malley AJ, Kakuhikire B, Perkins JM, et al. The social network context of HIV stigma: population-based, sociocentric network study in rural Uganda. Soc Sci Med. 2019;1(233):229-36.

17. Stein JH, Korcarz CE, Hurst RT, Lonn E, Kendall CB, Mohler ER, et al. Use of carotid ultrasound to identify subclinical vascular disease and evaluate cardiovascular disease risk: a consensus statement from the American Society of Echocardiography Carotid Intima-Media Thickness Task Force Endorsed by the Society for Vascular. J Am Soc Echocardiogr. 2008;21(2):93-111.
18. Korcarz CE, Hirsch AT, Bruce C, DeCara JM, Mohler ER, Pogue B, et al. Carotid intima-media thickness testing by non-sonographer clinicians: the office practice assessment of carotid atherosclerosis study. J Am Soc Echocardiogr. 2008s;21(2):117-22.

19. Fritz HF, Jutzy RV, Bansal R, Housten-Feenstra L. Validation of an automated computerized analyzing system for measuring common carotid artery intima-media thickness by brightness mode ultrasound. J Vasc Ultrasound. 2005;29(1):21-6.

20. Touboul P-J, Hennerici MG, Meairs S, Adams H, Amarenco P, Bornstein N, et al. Mannheim carotid intima-media thickness consensus (2004-2006). An update on behalf of the Advisory Board of the 3rd and 4th Watching the Risk Symposium, 13th and 15th European Stroke Conferences, Mannheim, Germany, 2004, and Brussels, Belgium, 2006. Cerebrovasc Dis Basel Switz. 2007;23(1):75-80.

21. American Heart Association. Body Mass Index (BMI) In Adults | American Heart Association [Internet]. 2014. Available from: https://www.heart.org/ en/healthy-living/healthy-eating/losing-weight/bmi-in-adults

22. Hagstromer M, Oja P, Sjostrom M. The International Physical Activity Questionnaire (IPAQ): a study of concurrent and construct validity. Public Health Nutr. 2006;1 (9):755-62.

23. Consolidated Guidelines on the Use of Antiretroviral Drugs for Treating and Preventing Hiv Infection: Recommendations for a Public Health Approach. [Internet]. 2016. Available from: https://www.deslibris.ca/ID/ 10089566

24. Godoi ETAM, Brandt CT, Lacerda HR, Godoi JTAM, de Oliveira DC, Costa GFAS, et al. Intima-media thickness in the carotid and femoral arteries for detection of arteriosclerosis in human immunodeficiency virus-positive individuals. Arq Bras Cardiol. 2017;108(1):3-11.

25. Janjua SA, Staziaki PV, Szilveszter B, Takx RAP, Mayrhofer T, Hennessy $\mathrm{O}$, et al. Presence, characteristics, and prognostic associations of carotid plaque among people living with HIV. Circ Cardiovasc Imaging. 2017;10(10):1-9.

26. Hsue PY, Lo JC, Franklin A, Bolger AF, Martin JN, Deeks SG, et al. Progression of atherosclerosis as assessed by carotid intima-media thickness in patients with HIV infection. Circulation. 2004;109:1603-8.

27. Nguyen D, Stanek V, Guillemi S, Ding E, Colley G, Montaner J, et al. High prevalence of carotid plaque identified using standard carotid ultrasound techniques in HIV-positive patients with low to moderate vascular risk. J AIDS Clin Res. 2014;5:9.

28. Stacey AR, Norris PJ, Qin L, Haygreen EA, Taylor E, Heitman J, et al. Induction of a striking systemic cytokine cascade prior to peak viremia in acute human immunodeficiency virus type 1 infection, in contrast to more modest and delayed responses in acute hepatitis $B$ and $C$ virus infections. J Virol. 2009:83(8):3719-33.

29. Alcaide ML, Rodriguez VJ, Abbamonte JM, Pallikkuth S, Langlie J, Parrish MS, et al. HIV and carotid atherosclerosis: a mediational model. AIDS Care 2020;32(7):907-11.

30. Kamtchum-Tatuene J, Mwandumba HC, Mwangalika Kachingwe G, Bonnett $\sqcup$, Kayange N, Solomon T, et al. A cross-sectional feasibility study of neurovascular ultrasound in Malawian adults with acute stroke-like syndrome. PLoS ONE [Internet]. 2020 Feb 7;15(2). Available from: https:// www.ncbi.nlm.nih.gov/pmc/articles/PMC7006928/

31. CDC. Heart Disease Risk Factors / cdc.gov [Internet]. 2015. Available from: https://www.cdc.gov/heartdisease/risk_factors.htm

32. Fryar CD, Chen T, Ph D, Li X. Prevalence of uncontrolled risk factors for cardiovascular disease. United States, 1999-2010. 2012;(103):1-8.

33. Ambrose JA, Barua RS. The pathophysiology of cigarette smoking and cardiovascular disease: an update. J Am Coll Cardiol. 2004;43(10):1731-7.

34. Woo SY, Joh JH, Han S-A, Park H-C. Prevalence and risk factors for atherosclerotic carotid stenosis and plaque: a population-based screening study. Medicine (Baltimore). 2017;96(4):e5999.

35. Mathiesen EB, Johnsen SH, Wilsgaard T, Bønaa KH, Løchen ML, Njølstad I. Carotid plaque area and intima-media thickness in prediction of firstever ischemic stroke: A 10-year follow-up of 6584 men and women: The Tromsø study. Stroke. 2011;

36. Spence JD, Eliasziw M, DiCicco M, Hackam DG, Galil R, Lohmann T. Carotid plaque area: a tool for targeting and evaluating vascular preventive therapy. Stroke. 2002;33(12):2916-22.

37. Brook RD. A negative carotid plaque area test is superior to other noninvasive atherosclerosis studies for reducing the likelihood of having 
underlying significant coronary artery disease. Arterioscler Thromb Vasc Biol. 2005;26(3):656-62.

\section{Publisher's Note}

Springer Nature remains neutral with regard to jurisdictional claims in published maps and institutional affiliations.

- fast, convenient online submission

- thorough peer review by experienced researchers in your field

- rapid publication on acceptance

- support for research data, including large and complex data types

- gold Open Access which fosters wider collaboration and increased citations

- maximum visibility for your research: over 100M website views per year

At BMC, research is always in progress.

Learn more biomedcentral.com/submissions 